\title{
CONTROLE DE TORTRICÍDEOS EM MACIEIRA COM DUAS FORMULAÇÕES DE Bacillus thuringiensis VAR. kustaki EM FRAIBURGO-SC ${ }^{1}$
}

\author{
LINO BITTENCOURT MONTEIRO² \& ALEXANDER SOUZA
}

RESUMO - Grapholita molesta e Bonagota cranaodes são duas importantes pragas de pomares de macieira controlada por inseticidas. O objetivo deste estudo foi testar duas formulações de Bacillus thuringiensis var. kurstaki (Btk) para o controle desses tortricídeos. Parcelas de macieira 'Fuji' foram pulverizadas com Dipel PM e Dipel SC, nas concentrações de $100 \mathrm{~mL}$ por100 L de calda. A eficiência do Btk foi comparada com os inseticidas tebufenozide (Mimic $240 \mathrm{SC}-90 \mathrm{ml}$ por $100 \mathrm{~L}$ ) e clorpyrifos (Lorsban $480 \mathrm{BR}-150 \mathrm{~mL}$ por $100 \mathrm{~L}$ ). Duas vezes por semana, eram avaliadas as capturas de pragas em armadilha do tipo Delta. As avaliações de danos nos frutos foram realizadas antes e na colheita, sendo classificados os frutos em função do agrupamento em cachopa e a sua localização na planta. Os tratamentos com Btk tiveram mais danos do que os químicos. Houve uma tendência de maiores danos na parte interna, próximo ao tronco das macieiras e o agrupamento dos frutos não influenciou na presença de danos. Ambas as formulações de Dipel foram eficientes quando comparados com Mimic e Lorsban.

Termos para indexação: Grapholita molesta, Bonagota cranaodes, macieira, entomopatógeno.

\section{TORTRICID MOTH CONTROL IN APPLEWITH TWO FORMULATION OF Bacillus thuringiensis VAR. kustaki IN FRAIBURGO, SC}

\begin{abstract}
Grapholita molesta and Bonagota cranaodes are two key pests in apple orchards controlled by insecticides. The objective of the present study was to test two formulas of Bacillus thuringiensis var. kurstaki to control these tortricid moths. A plot of 'Fuji' apple trees were sprayed with Dipel PM and Dipel $\mathrm{SC}$, at a concentration of $100 \mathrm{~mL}$ per 1001 liquid. The efficiency of Btk was compared with the insecticides tebuphenozide (Mimic $240 \mathrm{SC}-90 \mathrm{~mL}$ per $100 \mathrm{~L}$ ) and chlorpyrifos (Lorsban $480 \mathrm{BR}-150 \mathrm{~mL}$ per $100 \mathrm{~L}$ ). The capture of pests in a Delta-type trap was assessed twice a week. Fruit damage was evaluated pre- and post- harvest and the fruit were classified by branch-end clustering and location on the plant. The treatments with $B$. thuringiensis were more damaged than those with chemical treatments. There were a tendency to have more damage in the internal part, close to the apple tree trunk and the fruit clustering did not influence damage occurrence. Both Dipel formulations were efficient in relation of Mimic and Lorsban.
\end{abstract}

Index terms: Grapholita molesta, Bonagota cranaodes, apple tree, Bacillus.

\section{INTRODUÇ̃̃O}

Na cultura da macieira, diversas lagartas podem provocar diferentes graus de danos econômicos (FONSECA, 2006); entretanto, as da família Tortricidae são as que provocam os maiores prejuízos econômicos. No Brasil, há 800 espécies de lagartas dessa família (BROWN;FREITAS, 1999), sendo que em macieira ocorrem Grapholita molesta (Busck) e Bonagota cranaodes (Meyrick) (Lepidoptera: Tortricidae), respectivamente, originadas da Ásia e América do Sul. Além dessas, mundialmente ocorre Cydia pomonella (L.) (Lepidoptera: Tortricidae), entretanto não está presente em pomares comerciais na região de Fraiburgo -SC.
O controle de lagartas em macieira é realizado com inseticidas dos grupos organofosforado e regulador de crescimento, entretanto a Produção Integrada (PROTAS;SANHUEZA, 2004) limita o uso de alguns ingredientes ativos. $\mathrm{O}$ uso repetitivo de inseticidas no mesmo ciclo vegetativo pode selecionar populações resistentes (SAUPHANOR et al., 1998; IORIATTI et al., 2007), comprometendo a eficiência do controle.

Agentes biológicos entomopatógenos são uma opção ainda pouco explorada, em função da sensibilidade desses aos raios ultravioleta e baixa persistência (HABIB; ANDRADE, 1998). No entanto, representam um potencial de uso em programas de controle biológico e produção

'(Trabalho 140-09). Recebido em: 04-06-2009. Aceito para publicação: 22-02-2010.

2Prof. Dr. da Universidade Federal do Paraná, Cx. Postal 19061, CEP 81831-990, Curitiba-PR. E-mail: 1bmonteiro@terra.com.br ${ }^{3}$ Eng. Agrônomo da Agrícola Fraiburgo, Rod. Das Maçãs, 453, km 50, 89560-000, Fraiburgo-SC. 
integrada (DIBTANTORO;SISWOJO, 1988), pois deixam poucos resíduos e causam pouco impacto sobre inimigos naturais e meio ambiente. $\mathrm{O}$ uso de Bacillus thuringiensis, associado a uma estratégia de manejo integrado, pode promover controle de pragas nos períodos em que o controle químico é limitado pelo período de carência, próximo à colheita, ou pela presença de abelhas na floração (GRAFTONCARDWELL et al., 2005).

O objetivo deste estudo foi de verificar a eficiência de duas formulações à base de $B$. thuringiensis var. kurstaki em G. molesta e $B$. cranaodes em pomares comerciais de macieira, em Fraiburgo-SC, relacionando o status dos frutos nas plantas.

\section{MATERIAIS E MÉTODOS}

Delineamento experimental. $O$ experimento foi realizado em um pomar comercial de macieira 'Fuji' de um ha, plantado em 1985 no espaçamento 1,5 m entre plantas e 4,5 m entre linhas, pertencente à Agrícola Fraiburgo S/A, Fraiburgo-SC. As plantas foram conduzidas no formato de eixo central, com quatro ramos secundários distribuídos em dois níveis. O experimento foi conduzido no delineamento inteiramente casualizado, constituído de cinco blocos, cada um contendo cinco linhas de plantio. Cada bloco foi formado por nove macieiras em sequência e localizadas em cada uma das três linhas de plantio centrais, nas quais foram realizadas as avaliações de danos de pragas. A bordadura foi formada por quatro macieiras nas cinco linhas de plantio e pelas duas fileiras de plantas restantes.

Tratamentos. Foram avaliados dois inseticidas à base de Bacillus thuringienis var. kurstaki (Btk), marca comercial Dipel (Abbott Laboratórios do Brasil), nas formulações pó molhável (Dipel PM) e suspensão concentrada (Dipel SC), respectivamente, contendo 16.000 e 17.600 unidades internacionais de potência por mg (UIP), ambos testados nas concentrações de $100 \mathrm{~mL}$ por $100 \mathrm{~L}$ de calda. A eficiência do Btk foi comparada com os inseticidas tebufenozide (Mimic $240 \mathrm{SC}-90 \mathrm{~mL}$ por $100 \mathrm{~L}$ ) e clorpyrifos ethyl (Lorsban $480 \mathrm{BR}-150 \mathrm{~mL}$ por $100 \mathrm{~L}$ ) (Dow AgroSciences). Os inseticidas químicos foram misturados com um espalhante adesivo, marca Ag-Bem (Rohm and Haas Brasil), na concentração de $300 \mathrm{~mL}$ por ha, sendo que, na testemunha, foi aplicado água e Ag-Bem. Foi pulverizado o equivalente a $1.000 \mathrm{~L}$ de calda por hectare, utilizando um micropulverizador Pulmac 130, cuja vazão foi de $0,7 \mathrm{~L}$ de calda por macieira, aplicada em aproximadamente 45 segundos, com igual vazão para todos os tratamentos, mediante prévia calibragem do pulverizador. As pulverizações foram iniciadas às $16 \mathrm{~h}$ nas datas: $21-12$ e $29-12-$ 2000; 5-01 e 13-01-2001; 9-02 e 20-02-2001, de acordo com o monitoramento dos insetos. O nível de controle foi definido em 20 indivíduos para cada espécie por armadilha/semana. Em 20-11-2000, foi empregado um inseticida em todo o pomar com fenitrotion (Sumithion $500 \mathrm{CE}-150 \mathrm{ml}$ por $100 \mathrm{~L}$ ), necessário para o controle de Anastrepha fraterculus (Wied) (Diptera: Tephritidae).

Monitoramento das pragas. $\mathrm{Na}$ parcela experimental, foi instalada uma armadilha do tipo Delta com feromônios da BioControle (São Paulo) e IscaTecnologia (Ijuí), instaladas a 1,8 $\mathrm{m}$ de altura, respectivamente, para o monitoramento de $G$. molesta e $B$. cranaodes. Os feromônios foram trocados em intervalos de 45 dias. Duas vezes por semana, eram avaliadas as capturas de pragas, as quais definiram as datas de pulverização dos inseticidas. Próximo à colheita, não houve tratamento em função do período de carência de cada produto.

Avaliação de danos. As avaliações foram realizadas nos dias 3-01, 9-01 e 23-01-2001; 7-02 e $21-02-2001 ; 12-03$ e 30-03-2001 (colheita). Os frutos foram classificados em função do agrupamento em cachopa, definidos em três categorias: frutos desenvolvendo-se isolados na planta, dois frutos desenvolvendo-se no mesmo buquê floral e três frutos. Além disso, as avaliações sobre os frutos levaram em consideração à localização na planta em que eles se desenvolveram: zona 1- situa-se na parte superior da planta acima de 2,1 $\mathrm{m}$ até 3,50 $\mathrm{m}$ de altura em relação ao solo; zona 2 - compreendida por uma área externa da planta de $0,40 \mathrm{~m}$ a 2,1 $\mathrm{m}$ de altura; zona 3 - situa-se no interior da copa, em formato triangular e limitada pela região 2 (Figura 1). Todas as avaliações anteriores à colheita foram visuais, e os frutos danificados foram retirados da planta. $\mathrm{Na}$ colheita, os frutos foram retirados e armazenados em caixas plásticas para avaliação do dano de $B$. cranaodes e G. molesta. A eficiência dos produtos foi calculada pela fórmula: mortalidade na testemunha $(\%)$; mortalidade no tratamento $(\%) /$ mortalidade na testemunha (\%)x100 (Abbott, 1925). Os dados foram submetidos à análise de variância, e as médias, comparadas pelo teste de Tukey $(\mathrm{p}<0,05)$, analisados pelo programa Statgraphics Plus 4.1.

\section{RESULTADOS E DISCUSSÃO}

O potencial de dano de G. molesta e B. cranaodes foi avaliado a partir do monitoramento capturas de machos (Figura 2), norteando as decisões de pulverização. A primeira aplicação de 
inseticida ocorreu quando as capturas de G. molesta estavam baixas, entretanto essa decisão baseou-se em função da última pulverização ter ocorrido há 31 dias (20-12). O pico populacional, a partir de 26-12, justificou as três pulverizações em intervalos de sete dias. A quinta pulverização ocorreu quando houve um acréscimo das capturas (27 dias após a quarta pulverização). A população de $G$. molesta voltou a ser importante com as capturas de 19-02, motivando o tratamento de 20-02.

No mesmo período, a flutuação populacional de $B$. cranaodes acompanhou a curva de capturas de $G$. molesta, entretanto o potencial de danos foi em torno de três vezes mais baixo. As pulverizações realizadas a partir da flutuação de $G$. molesta, provavelmente, tiveram ação sobre lagartas-enroladeiras.

O número de G. molesta capturadas no monitoramento foi capaz de causar danos significativamente diferentes entre os tratamentos e a testemunha $(\mathrm{d}=4, \mathrm{~F}=2,9842, \mathrm{p}=0,0199)$. Os tratamentos à base de $B$. thuringiensis tiveram, em média, cerca de três vezes mais danos do que os dois tratamentos quími$\cos$ (Tabelas 1 e 2), entretanto essa diferença não foi significativa. Seguindo Abbott (1923), Dipel PM para $B$. cranaodes foi mais eficiente do que tebufenozide (Tabela 2). Essa menor eficiência observada do $\mathrm{Bt}$ não foi constatada por Morandi Filho et al. (2007) em relação a inseticidas fosforados para o controle de Argyrotaenia sphaleropa (Meyrick) (Lepidoptera: Tortricidae). De forma geral, as formulações à base de Bacillus apresentam uma meia vida menor a campo do que as formulações químicas, pois os cristais proteicos ficam sobre as folhas submetidas à ação de raios ultravioleta. Estes degradam e esterilizam o agente biológico (MOÍNO JUNIOR, 2000), o que não ocorre, por exemplo, com tebufenozide. Este inseticida foi considerado eficiente no controle de $G$. molesta (GRÜTZMACHER et al., 1999), embora o ingrediente ativo necessite ser ingerido pelas lagartas como os Bts (GRAFTON-CARDWELL et al., 2005). $\mathrm{O}$ inseticida clorpyrifos possui ação de contato e ingestão, assim as lagartas não necessitam alimentarse para se intoxicarem, o que pode melhorar a ação tóxica em relação ao primeiro estágio de lagarta.

A formulação Dipel SC foi a que apresentou a menor eficiência de controle (ABBOTT, 1923) entre as duas formulações (Tabela 2), embora a concentração (UIP) na formulação SC seja maior. O resultado obtido pela formulação PM foi similar ao obtido por Dipel formulação em grãos dispersos (FD), realizados a campo por Morandi Filho et al. (2007).

Os prejuízos verificados por $B$. cranaodes foram diferentes entre os tratamentos e a testemunha $(\mathrm{d}=4, \mathrm{~F}=5,9083, \mathrm{p}=0,0001)$. As baixas capturas de B. cranaodes limitaram os danos, inferiores a $1 \%$ em todas as parcelas, e a eficiência de controle foi superior a $88 \%$. Não houve diferenças significativas entre os tratamentos (Tabela 1, 2). A formulação Dipel SC permitiu danos três vezes maiores do que o pó molhável, como aconteceu com $G$. molesta. Os tratamentos químicos tiveram eficiência de controle superior à obtida por Botton et al. (2000), mostrando a superioridade neste experimento em relação ao biológico.

Observou-se que os danos nas testemunhas foram semelhantes para ambas as pragas (Tabela 2 ). Pode-se considerar que o dano de $B$. cranaodes foi proporcionalmente maior que o realizado por G. molesta, em função da baixa captura média de lagarta-enroladeira durante o experimento. Isto sugere que os tratamentos foram mais eficientes para $G$. molesta do que para $B$. cranaodes. Danos de $8 \%$ obtidos neste experimento foram superiores aos mencionados por (KOVALESKI et al., 1998).

Analisando a localização dos danos nas macieiras em todos os tratamentos (Tabela 3), verifica-se que houve diferenças significativas para $B$. cranaodes $(\mathrm{d}=2, \mathrm{~F}=4,4307, \mathrm{P}=0,0129) \mathrm{e}$ G. molesta $(\mathrm{d}=2, \mathrm{~F}=3,8249, \mathrm{p}=0,0232)$. Quando se exclui a testemunha da análise, não se observa diferenças $(\mathrm{d}=2, \mathrm{~F}=2,8054, \mathrm{p}=0,0631)$. A zona 3 (inferior e interior da planta) foi onde ocorreram as maiores porcentagens de danos em ambas as pragas. A redução da eficiência dos inseticidas nessa zona pode estar relacionada com a tecnologia de pulverização, pois as macieiras foram conduzidas de modo que o ramo secundário se desenvolve perpendicularmente à linha de plantio (condução de quatro ramos secundários e dois níveis). Esse tipo de condução pode impedir a penetração e a distribuição eficiente da calda inseticida no interior da planta. Quando se analisam os danos obtidos na testemunha (Tabela 1), portanto sem a influência de inseticida, verificase que a zona 3 teve significativamente mais frutos danificados em relação aos obtidos nas zonas 1 e 2, sugerindo que ambas as pragas tenham preferência em desenvolverem-se na zona 3.

Os danos ocorridos em frutos agrupados não tiveram diferença significativa. Os frutos isolados apresentaram uma tendência de possuírem mais danos do que os frutos agrupados, principalmente para G. molesta (Tabelas 1 e 3). Na prática, os produtores relatam que $B$. cranaodes provoca mais danos em frutos desenvolvidos em cachopas (agrupamento de dois e três frutos). Isso poderia ser pela redução da penetração de inseticidas no pedúnculo dos frutos, local onde frequentemente se instalam as lagartasenroladeiras (KOVALESKI et al., 1998), o que não se verificou neste experimento. A distribuição dos danos na testemunha foi uniforme em quase todos os três agrupamentos de frutos. 
TABELA 1 - Danos em maçãs (\%) de Grapholitas molesta e Bonagota cranaodes cranaodes de acordo com a localização do fruto na planta e agrupamento do fruto, em quatro tratamentos de inseticidas, Fraiburgo, 2000-01.

\begin{tabular}{lcccccccccccc}
\hline \multirow{2}{*}{ Praga } & \multicolumn{2}{c}{ Dipel PM } & \multicolumn{2}{c}{ Dipel SC } & \multicolumn{2}{c}{ Mimic } & \multicolumn{2}{c}{ Lorsban } & \multicolumn{2}{c}{ Testemunha } \\
\cline { 2 - 12 } & & Planta $^{1}$ & Fruto $^{2}$ & Planta & Fruto & Planta & Fruto & Planta & Fruto & Planta & Fruto \\
\hline Bonagota & 1 & $0,1 \mathrm{a}$ & $0,5 \mathrm{a}$ & $1,1 \mathrm{a}$ & $1,6 \mathrm{a}$ & $0,8 \mathrm{a}$ & $0,6 \mathrm{a}$ & $0,2 \mathrm{a}$ & $0,1 \mathrm{a}$ & $1,7 \mathrm{a}$ & $11,4 \mathrm{a}$ \\
& 2 & $0,3 \mathrm{a}$ & $0,5 \mathrm{a}$ & $1,0 \mathrm{a}$ & $1,4 \mathrm{a}$ & $0,7 \mathrm{a}$ & $1,1 \mathrm{a}$ & $0,2 \mathrm{a}$ & $0,3 \mathrm{a}$ & $1,0 \mathrm{a}$ & $3,0 \mathrm{a}$ \\
& 3 & $0,5 \mathrm{a}$ & $0,0 \mathrm{a}$ & $0,9 \mathrm{a}$ & $0,0 \mathrm{a}$ & $0,6 \mathrm{a}$ & $0,4 \mathrm{a}$ & $0,0 \mathrm{a}$ & $0,0 \mathrm{a}$ & $23,6 \mathrm{~b}$ & $11,8 \mathrm{a}$ \\
& & & & & & & & & & & & \\
Grapholita & 1 & $0,7 \mathrm{a}$ & $3,0 \mathrm{a}$ & $1,8 \mathrm{a}$ & $5,4 \mathrm{a}$ & $0,3 \mathrm{a}$ & $0,2 \mathrm{ab}$ & $0,8 \mathrm{a}$ & $2,3 \mathrm{a}$ & $2,4 \mathrm{a}$ & $8,9 \mathrm{a}$ \\
& 2 & $0,9 \mathrm{a}$ & $0,6 \mathrm{a}$ & $1,1 \mathrm{a}$ & $1,7 \mathrm{a}$ & $0,1 \mathrm{a}$ & $1,0 \mathrm{~b}$ & $0,2 \mathrm{a}$ & $0,1 \mathrm{a}$ & $1,3 \mathrm{a}$ & $7,5 \mathrm{a}$ \\
& 3 & $2,0 \mathrm{a}$ & $0,0 \mathrm{a}$ & $4,1 \mathrm{a}$ & $0,0 \mathrm{a}$ & $0,8 \mathrm{a}$ & $0,0 \mathrm{a}$ & $1,3 \mathrm{a}$ & $0,0 \mathrm{a}$ & $20,0 \mathrm{~b}$ & $7,3 \mathrm{a}$ \\
\hline
\end{tabular}

Médias seguidas de mesma letra minúscula não diferem entre si na coluna, para cada praga, pelo teste de Tukey $(\mathrm{P}<0,005)$ ${ }^{1}$ Localização do fruto na planta: $1=$ fruto situado a mais do que $2,1 \mathrm{~m}$ de altura; 2 = fruto situado entre 0,4 e $2,1 \mathrm{~m}$ na parte exterior da planta; 3 = fruto situado entre 0,4 e $1,7 \mathrm{~m}$ no interior da planta e delimitada pela localização ' 2 '.

${ }^{2}$ Frutos agrupados em cachopa de $1 ; 2$ e 3 frutos.

TABELA 2 - Danos gerais em maçã (\%) e eficiência de quatro inseticidas sobre Bonagota cranaodes e Grapholitas molesta, Fraiburgo-SC, 2001-01.

\begin{tabular}{lcccc}
\hline \multirow{2}{*}{ Tratamento } & \multicolumn{2}{c}{ B. cranaodes } & \multicolumn{2}{c}{ G. molesta } \\
\cline { 2 - 5 } & Danos (\%) & Eficiência $^{1}$ & Danos (\%) & Eficiência \\
\hline Dipel PM & $0,3 \pm 0,22$ a & 96,5 & $1,2 \pm 0,71$ a & 84,8 \\
Dipel SC & $1,0 \pm 0,35$ a & 88,5 & $2,3 \pm 1,15 \mathrm{a}$ & 70,8 \\
Mimic & $0,7 \pm 0,28$ a & 91,9 & $0,4 \pm 0,21 \mathrm{a}$ & 94,9 \\
Lorsban & $0,1 \pm 0,08$ a & 98,8 & $0,7 \pm 0,48 \mathrm{a}$ & 91,1 \\
Testemunha & $8,7 \pm 3,35 \mathrm{~b}$ & & $7,9 \pm 3,73 \mathrm{~b}$ & \\
\hline
\end{tabular}

Médias seguidas de mesma letra minúscula não diferem entre si na coluna,pelo testede Tukey $(\mathrm{P}<0,05)$.

${ }^{1}$ Eficiência do controle (\%) baseada em Abbott (1923).

TABELA 3 - Danos em maçã (\%) de Bonagota cranaodes e Grapholitas molesta em função do agrupamento de frutos e a sua localização na planta Fraiburgo-SC, 2000-01.

\begin{tabular}{|c|c|c|c|c|c|c|}
\hline \multirow{3}{*}{ Localização $^{1}$} & \multicolumn{6}{|c|}{ Praga / Frutos agrupados ${ }^{2}$} \\
\hline & \multicolumn{3}{|c|}{ B. cranaodes } & \multicolumn{3}{|c|}{ G. molesta } \\
\hline & 1 & 2 & 3 & 1 & 2 & 3 \\
\hline 1 & $0,6 \mathrm{aA}$ & $0,7 \mathrm{aA}$ & $1,0 \mathrm{aA}$ & $2,2 \mathrm{bA}$ & $1,0 \mathrm{abA}$ & $0,4 \mathrm{aA}$ \\
\hline 2 & $0,8 \mathrm{bA}$ & $1,0 \mathrm{bA}$ & $0,0 \mathrm{aA}$ & $1,3 \mathrm{bA}$ & $0,8 \mathrm{abA}$ & $0,0 \mathrm{aA}$ \\
\hline 3 & $7,0 \mathrm{aA}$ & $2,0 \mathrm{aA}$ & $6,3 \mathrm{aA}$ & $8,2 \mathrm{aA}$ & $4,8 \mathrm{aA}$ & $4,0 \mathrm{aA}$ \\
\hline
\end{tabular}

Média seguida de mesma letra minúscula não difere entre si na linha, para cada praga, e maiúscula na coluna, pelo teste de Tukey $(\mathrm{P}<0,005)$.

${ }^{1}$ Localização do fruto na planta: $1=$ fruto situado a mais do que $2,1 \mathrm{~m}$ de altura; $2=$ fruto situado entre 0,4 e $2,1 \mathrm{~m}$ na parte exterior da planta; 3 = fruto situado entre 0,4 e 1,7 m no interior da planta e delimitada pela localização ' 2 '.

${ }^{2}$ Frutos agrupados em cachopa de 1; 2 e 3 frutos. 


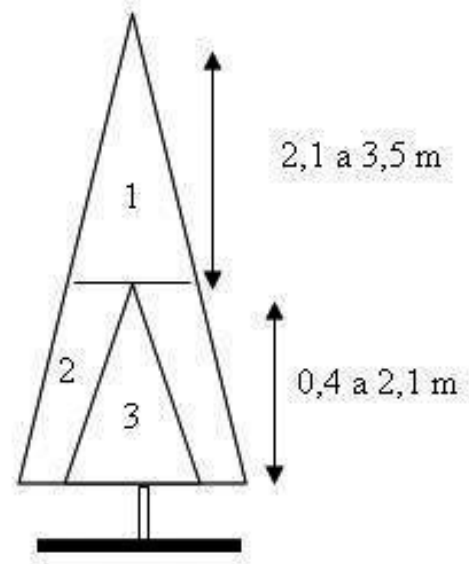

FIGURA 1 - Zonas de macieira (1; 2 e 3) para avaliar danos em frutos, causados por Grapholitas molesta e Bonagota cranaodes.

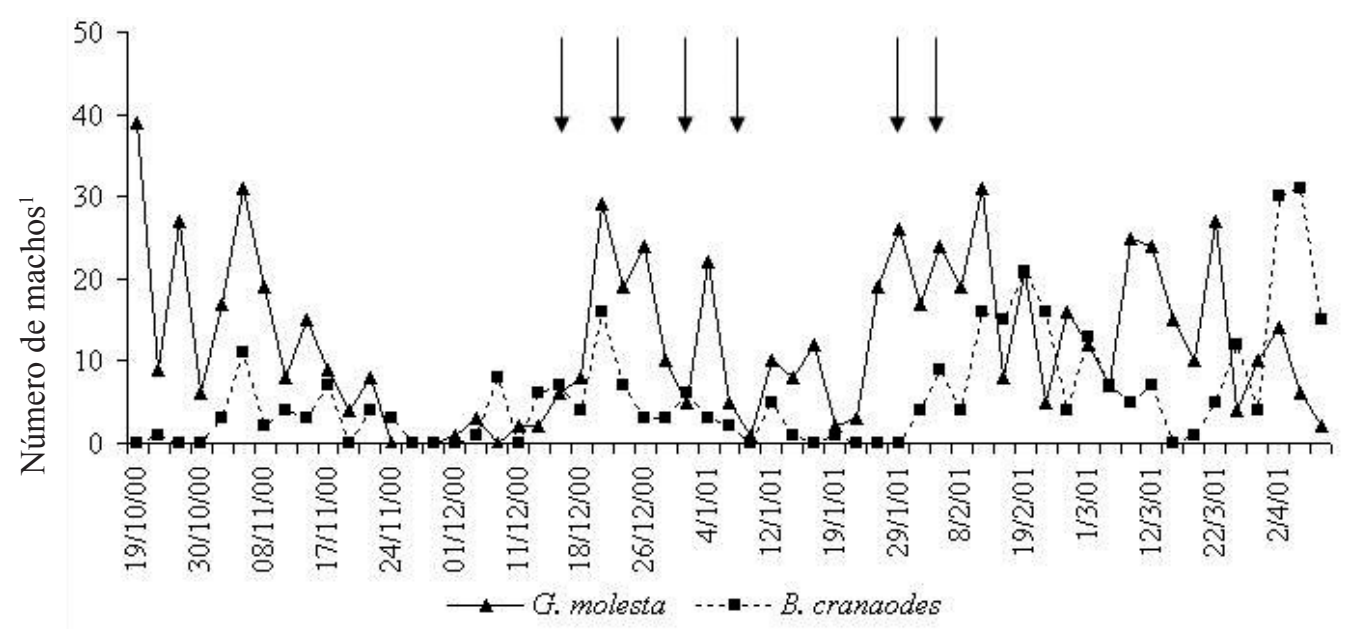

FIGURA 2 - Captura de G. Molesta e Bonagota Cranaodes com armadilha de feromônio dfo tipo Delta, em pomar de macieira. Setas indicam as datas de tratamento de inseticidas. Fraiburgo, 2001-01.

${ }^{1}$ Número de machos por dia de amostragem

\section{CONCLUSÃO}

As formulações à base de Bacillus thuringiensis var. kurstaki é tão eficientes quanto tebufenozide e clorpyrifos para o controle de Grapholitas molesta e Bonagota cranaodes. Os danos são influenciados pela localização do fruto na macieira e os frutos localizados nas partes inferior e interior da planta (zona 3) são os que apresentaram maiores danos.

\section{REFERÊNCIAS}

ABBOTT, W.S. A method of computing the effectiveness of an insecticide. Journal of Economic Entomology, Lanham, v.18, p.265-267, 1925.

BOTTON, M; NAKANO, O; KOVALESKI, A. Controle químico da lagarta-enroladeira (Bonagota cranaodes Meyrick) na cultura da macieira. Pesquisa Agropecuária Brasileira, Brasília, 35; p. 2139-2144, 2000. 
BROWN JUNIOR, K. S.; Freitas A.V.L. Lepidoptera. In: C. A. JOLY; C. E. M. Bicudo. (Org.). Biodiversidade do Estado de São Paulo, Brasil: síntese do conhecimento ao final do século XX - Invertebrados Terrestres - BRANDÃO, C. R. F. ; Cancello, E. M. (Eds.). FAPESP, São Paulo, 1999, p.225-243.

DIBTANTORO, A. Z. H; SISWOJO S. Approch to integrated control of some vegetable insects-pests by using microbial insecticides Bacillus thuringiensis. Bull. Penelit. Hortic., Jacarta, 16; p.67-72, 1988.

FONSECA, F. L. Ocorrência, monitoramento, caracterização de danos e parasitismo de noctuidae e geometridae em pomares comerciais de macieira em Vacaria, Rio Grande do Sul, Brasil. UFPR, 80p, Tese de doutorado, 2006.

GRAFTON-CARDWELL, E.; EGODFREY, L. D.; CHANEY, W. E.; BENTLEY, W. J. Various novel insecticides are less toxic to humans, more specific to key pests. California Agriculture, Davis, v.59: 29-34, 2005.

GRÜTZMACHER, A. D.; LOECK, A.E.; FACHINELLO, J.C.; GRÜTZMACHER, D.D.; GARCIA, M.S. Eficiência dos inseticidas fisiológicos Mimic $240 \mathrm{sc}$ (tebufenozide) e Intrepid 240 sc (methoxifenozide) no controle da mariposa oriental Grapholita molesta (busck, 1916) (Lepidoptera: Tortricidae) na cultura da pereira. Revista Brasileira Agrociência, Santa Maria, 5; p.211-215, 1999.

HABIB, M.E.M; ANDRADE, C.F.S. Bactérias entomopatogênicas. In. Controle microbiano de inseto, Sérgio Batista Alves Ed., 2. ed, FEALQ, Piracicaba, 1998. p. 383-445
IORIATTI C.; TASIN, M.; CHARMILLOT, P.J.; REYES, M.; SAUPHANOR, B. Early detection of resistance to tebufenozide in field populations of Cydia pomonella L.: methods and mechanisms. Journal Applied Entomology, 131: 453-459, 2007.

KOVALESKI, A.; BOTTON, M.; EIRAS, A.E.; VILELA, E. Lagarta-enroladeira da macieira Bonagota cranaodes (Meyrick, 1937) (Lepidoptera: Tortricidae): Bioecologia, monitoramento e controle. Bento Gonçalves: Embrapa Uva e Vinho, 16 p. (Circular Técnica, 24), 1998.

MOÍNO JUNIOR, A. Produção de fungos, vírus e bactérias entomopatogênicas. In. Vanda H. P. Bueno (Ed.), Controle Biológico de Pragas: Produção massal e controle de qualidade. Lavras, 2000, $\mathrm{p}: 175-186$.

MORANDI FILHO W.J.; BOTTON, M.; GRÜTZMACHER, A.D.; ZANARDI, O.Z. Efeito de Bacillus thuringiensis e inseticidas químicos no controle de Argyrotaenia sphaleropa (Meyrick, 1909) (Lepidoptera: Tortricidae) em Videira. Arquivos Instituto Biologia, Campinas, 74; p. 129-134, 2007.

PROTAS, J. F. S.; SANHUEZA, R.M. Normas técnicas e documentos de acompanhamento da produção integrada de maçã $-2^{\circ}$ versão. Bento Gonçalves-RS: Embrapa Uva e Vinho, 2004. 80 p (Documentos, 33), 2004

SAUPHANOR, B.; BOUVIER, J.C.; BROSSe, V. Spectrum of Insecticide Resistance in Cydia pomonella (Lepidoptera: Tortricidae) in Southeastern France. Journal Economic Entomology, Lanham, v.91; p.1225-1231, 1998 